\title{
Die Umsetzung von EU-Richtlinien im Zeichen der Parteipolitik: Eine akteurszentrierte Antwort auf die Misfit-These
}

\section{Oliver Treib}

Vor dem Hintergrund der Debatte über die Steuerungsfähigkeit der EU befasst sich dieser Beitrag mit der Frage, welche Faktoren zu einer korrekten und pünktlichen Umsetzung von EU-Richtlinien beitragen. Er setzt sich mit der häufig vertretenen Misfit-These auseinander, der gemäß Qualität und Schnelligkeit der nationalen Anpassung vor allem durch das Ausmaß der Kompatibilität zwischen europäischen Vorgaben und bestehenden nationalen Strukturen bestimmt wird. Anhand empirischer Ergebnisse über die Umsetzung von sechs arbeitsrechtlichen Richtlinien in vier Ländern wird die begrenzte Erklärungskraft dieser These demonstriert. Der Beitrag weist stattdessen auf die zentrale Rolle der nationalen Parteipolitik hin und diskutiert die Implikationen dieses Befundes für die Problemlösungskapazität der EU.

\section{Einleitung ${ }^{1}$}

In den letzten Jahren ist viel über die „Problemlösungsfähigkeit“ der europäischen Politik diskutiert worden (siehe vor allem Scharpf 1998, 1999; Grande/Jachtenfuchs 2000). Kann die Europäische Union dazu beitragen, drängende gesellschaftliche Probleme wie die anhaltende Massenarbeitslosigkeit, die Modernisierung der Rentensysteme angesichts der Überalterung der Gesellschaft oder die zunehmende Verknappung natürlicher Ressourcen zu lösen? Oder sollte man seine Hoffnungen diesbezüglich eher auf die verbleibenden Handlungsressourcen des Nationalstaats richten? Die wissenschaftliche Debatte hierüber konzentrierte sich bislang hauptsächlich auf die Frage, ob die verschiedenen Entscheidungsverfahren der Europäischen Union angesichts der zugrunde liegenden Interessenkonstellationen nationaler und supranationaler Akteure in der Lage sind, Entscheidungen zu produzieren, mit denen bestimmte für wichtig gehaltene Ziele potenziell verwirklicht werden könnten (Falkner 2000).

1 Das empirische Material dieses Beitrags wurde im Rahmen eines Projektverbundes über „Neues Regieren und soziales Europa: $\mathrm{Zu}$ Theorie und Praxis von Mindestharmonisierung und Soft Law im europäischen Mehrebenensystem" unter Leitung von Gerda Falkner am Max-PlanckInstitut für Gesellschaftsforschung erarbeitet. Zu Zwischenergebnissen des Projektverbundes siehe http://www.mpi-fg-koeln.mpg.de/socialeurope. Für hilfreiche Kommentare und Anregungen bedanke ich mich bei Gerda Falkner, Armin Schäfer, Désirée Schauz, Patrick Ziltener sowie den beiden anonymen Gutachtern der PVS. Des Weiteren danke ich Wolfgang Wessels für die wissenschaftliche Begleitung und Unterstützung meines Dissertationsprojekts (Treib 2002), das die Grundlage dieses Papiers bildet. 
Auffallend ist, dass dem Aspekt der Implementation europäischer Regelungen in dieser Diskussion bisher vergleichsweise wenig Aufmerksamkeit geschenkt wurde. Dies muss erstaunen, ist es doch für die Beurteilung der Gestaltungsfähigkeit der supranationalen Politik von zentraler Bedeutung, inwiefern die EU sicherstellen kann, dass die Ziele der in Brüssel verabschiedeten verbindlichen Rechtsakte auch tatsächlich in praktische Politik umgewandelt werden. Können potenziell problemadäquate europäische Regelungen überhaupt ihre Steuerungswirkung entfalten oder scheitert die Problemlösung an signifikanten Implementationsdefiziten? Diese Frage erscheint umso wichtiger, wenn man bedenkt, dass die Union über keinen eigenen Verwaltungsunterbau zur Durchführung ihrer Rechtsakte verfügt, sondern diesbezüglich größtenteils auf die Kooperation der Mitgliedsstaaten angewiesen ist. In dieser Hinsicht ähnelt das europäische Mehrebenensystem sehr viel stärker dem deutschen Modell des Verbundföderalismus, bei dem die Gesetze der oberen Ebene durch die Verwaltungen der unteren Ebene implementiert werden, als dem System des dualen Föderalismus US-amerikanischer Prägung, wo jede Ebene ihre Gesetze durch jeweils eigene Verwaltungsbehörden durchführt (Scharpf 1985: 325).

Im Vergleich zur Durchführung von Bundesgesetzen im deutschen Föderalismus erscheint die Implementationsstruktur der EU jedoch besonders prekär: Bei der Implementation von EU-Richtlinien ist nicht nur der administrative Vollzug an die Verwaltungen der Mitgliedsstaaten delegiert, sondern auch noch ein Teil des Rechtsetzungsprozesses selbst. EU-Richtlinien geben lediglich Ziele vor, die von den Mitgliedsstaaten innerhalb einer bestimmten Frist in nationales Recht umgesetzt werden müssen, bevor sie dann von den Verwaltungen durchgeführt und von den gesellschaftlichen Adressaten angewendet werden können. Es tritt also ein zusätzlicher nationaler Rechtsetzungsprozess zwischen die Verabschiedung der europäischen Regelungen und ihre praktische Durchführung in den Mitgliedsstaaten. Die Notwendigkeit der Umsetzung fügt der dezentralen Implementationsstruktur der Europäischen Union daher einen weiteren entscheidenden "clearance point" hinzu (Pressman/Wildavsky 1973), an dem die praktische Verwirklichung der in den europäischen Rechtsakten niedergelegten Ziele scheitern kann.

Dass die Umsetzungsphase ein zentrales Einfallstor für Implementationsdefizite und somit für Steuerungsprobleme innerhalb der EU ist, zeigt bereits ein grober Blick auf die von der Europäischen Kommission eingeleiteten Verfahren gegen Mitgliedsstaaten, die ihre europarechtlichen Verpflichtungen nicht erfüllt haben. So bezogen sich rund zwei Drittel aller im Jahr 2000 versandten „mit Gründen versehenen Stellungnahmen“ und etwa ebenso viele der eingeleiteten Klageerhebungen vor dem EuGH auf Fälle fehlender oder mangelhafter Umsetzung von Richtlinien (KOM[2001] 309: Anhang I, Tabelle 2.2). Wenngleich sich in diesen Zahlen unentdeckt gebliebene Verstöße gegen europäisches Recht nicht widerspiegeln (Mendrinou 1996: 2), geben sie doch einen wichtigen Anhaltspunkt dafür, dass ein signifikanter Anteil von Implementationsproblemen innerhalb des europäischen Mehrebenensystems auf die Phase der Umsetzung zurückzuführen ist.

Aufgrund dieses Befundes steht die Analyse dieser Phase des Implementationsprozesses im Mittelpunkt des vorliegenden Beitrags: Inwiefern befolgen die Mitgliedsstaaten ihre europarechtlichen Verpflichtungen, indem sie die Vorgaben von EU-Richt- 
linien fristgerecht, vollständig und sachlich korrekt in nationales Recht überschreiben? Und welche Faktoren tragen zu einer effektiven und pünktlichen Umsetzung bei?

In der jüngeren Forschung findet sich vielfach das Argument, dass sich die Qualität und Schnelligkeit der nationalen Anpassung an europäische Vorschriften im Wesentlichen auf das Ausmaß der Kompatibilität oder Inkompatibilität zwischen europäischen Anforderungen und vorhandenen nationalen Regelungstraditionen zurückführen lässt: Verlangen die europäischen Vorgaben nur geringfügige Veränderungen der nationalen Arrangements, ist aus dieser Sicht eine unproblematische Implementation zu erwarten. Müssen jedoch signifikante Reformen an den bestehenden nationalen Regelungen vorgenommen werden, ist mit Widerständen und Verzögerungen oder mit gravierenden Implementationsmängeln zu rechnen (siehe insbesondere Duina 1997, 1999; Duina/ Blithe 1999; Knill/Lenschow 1998, 1999, 2000; Börzel 2000a, 2000b).

Dieser Misfit-These liegt die aus dem historischen bzw. soziologischen Institutionalismus abgeleitete theoretische Annahme zugrunde, dass bestehende institutionelle Arrangements im weitesten Sinne über eine erhebliche Beharrungskraft verfügen und daher schwer zu verändern sind (siehe etwa March/Olsen 1989; DiMaggio/Powell 1991; Thelen/Steinmo 1992; Immergut 1998; Pierson 2000). Die Interessen der Akteure auf der mitgliedsstaatlichen Ebene sind infolge dessen weit gehend durch die vorhandenen nationalen Strukturen determiniert: Es wird davon ausgegangen, dass nationale Regierungen, Verwaltungen und Parlamente bei der Umsetzung als „guardians of the status quo, as the shield protecting national legal-administrative traditions" agieren (Duina 1997: 157). Aus dieser Perspektive muss die grundsätzliche Steuerungsfähigkeit der EU skeptisch beurteilt werden. Selbst wenn es angesichts der heterogenen nationalen Interessen gelingen sollte, gemeinsame Beschlüsse auf der europäischen Ebene zu fassen, die mit den vorhandenen nationalen Ausgangsbedingungen im Widerspruch stehen, müsste deren Implementation auf der nationalen Ebene unweigerlich an den Beharrungskräften der existierenden Regelungsstrukturen scheitern.

Der vorliegende Beitrag konfrontiert diese vor allem auf Untersuchungen aus dem Bereich der Umweltpolitik basierende These mit Ergebnissen einer Studie über die Umsetzung von sechs arbeitsrechtlichen EU-Richtlinien in vier Mitgliedsstaaten. Die präsentierten empirischen Resultate lassen erhebliche Zweifel an der Erklärungskraft der Misfit-These aufkommen. Das Ausmaß der Passgenauigkeit zwischen europäischen Vorgaben und nationalen Regelungsbeständen ist nur in sehr begrenztem Maße in der Lage, die beobachteten Anpassungsmuster zu erklären. Daher stelle ich dieser auf strukturelle Inkompatibilitäten ausgerichteten Sichtweise eine akteurzentrierte Perspektive gegenüber, die auf die Eigenlogik nationaler politischer Prozesse und vor allem auf die zentrale Rolle politischer Akteure bei der Umsetzung von EU-Richtlinien abstellt. Dabei müssen die Interaktionen zwischen der für die Umsetzung verantwortlichen Regierung, den darin vertretenen Parteien, der Ministerialbürokratie und wichtigen Interessengruppen in den Blick genommen werden. Ein umfassender Überblick über das Funktionieren dieses Beziehungsgeflechts würde jedoch den Rahmen dieses Beitrags sprengen (siehe dazu ausführlich Treib 2002). Daher konzentriere ich mich hier auf den Einfluss eines Faktors, der sich in meiner empirischen Untersuchung als besonders wichtig erwiesen hat, bislang aber noch nicht systematisch in Betracht gezogen wurde: 
die Rolle von politischen Parteien und ihren Programmen bei der Umsetzung von EURichtlinien.

Meine Fallstudien liefern deutliche Hinweise darauf, dass Regierungen bei der Umsetzung nicht lediglich als Verteidiger des nationalen Status quo agieren, sondern europäische Vorgaben (auch) im Lichte ihrer parteipolitisch definierten Präferenzen beurteilen. Auf diese Weise können selbst weit reichende Reformerfordernisse ohne größere Probleme erfüllt werden, wenn sie mit den parteipolitischen Zielen der jeweiligen Regierung im Einklang stehen. Umgekehrt sind auch relativ geringfügige Anpassungen zum Scheitern verurteilt, wenn sie von der Regierung aus parteipolitischen Gründen abgelehnt werden. Dazu kommt, dass die Aktivierung parteipolitischer Unterstützung zu Überimplementation führen kann, d.h. dass die zuständige Regierung bei der Umsetzung über die erforderlichen Mindeststandards der Richtlinie hinausgeht. Durch das so entstehende zusätzliche Konfliktpotenzial kann es außerdem zu Verzögerungen kommen, die mit den verbindlichen Anpassungserfordernissen nicht zu erklären sind.

Der Beitrag ist wie folgt aufgebaut: Im folgenden Abschnitt (2) gebe ich einen Überblick über meine empirischen Ergebnisse und zeige, dass die Misfit-These nur in geringem Maße in der Lage ist, die beobachteten Anpassungsmuster zu erklären. Danach stelle ich mit Hilfe empirischer Beispiele verschiedene Formen des Einflusses der Parteipolitik auf die Umsetzung von EU-Richtlinien vor und diskutiere die beobachteten Effekte vor dem Hintergrund der Parteiendifferenz-These (3). Der letzte Abschnitt fasst die wesentlichen Ergebnisse des Beitrags zusammen, diskutiert Möglichkeiten und Grenzen der Verallgemeinerbarkeit dieser Resultate und weist auf ihre Implikationen für die generelle Steuerungsfähigkeit der EU hin (4).

\section{Die begrenzte empirische Erklärungskraft der Misfit-These}

Zur Überprüfung der Misfit-These habe ich 24 vergleichend angelegte Fallstudien über die Umsetzung von sechs Richtlinien in vier Ländern durchgeführt. Bei den ausgewählten Richtlinien handelt es sich um sechs der wesentlichen Rechtsakte, die in den 1990er Jahren im Rahmen der sozialen Dimension des europäischen Binnenmarktes auf EU-Ebene verabschiedet wurden. Diese Richtlinien betreffen die Gleichbehandlung von Teilzeitarbeitnehmern, das Recht auf Elternurlaub, die Festlegung von täglichen und wöchentlichen Höchstarbeitszeiten sowie von Jahresurlaubsansprüchen, den Schutz von Jugendlichen sowie von werdenden und stillenden Müttern bei der Arbeit und das Recht auf einen schriftlichen Nachweis über die wesentlichen Beschäftigungsbedingungen. ${ }^{2}$

Die Auswahl der Länder basiert auf der Überlegung, möglichst viel Varianz im Hinblick auf den zentralen Einflussfaktor der zu überprüfenden These, der Höhe des Anpassungsbedarfs, zu erhalten. Daher wurden mit Deutschland und den Niederlanden zwei kontinentale Länder ausgewählt, die bereits über ein hohes Niveau an Arbeitsmarktregulierung verfügen (Weiss 1995; Van der Heijden 1999), während das System gesetzlicher Arbeitnehmerrechte in den voluntaristisch geprägten Ländern

2 Richtlinien 97/81/EG, 96/34/EG, 94/33/EG, 93/104/EG, 92/85/EWG und 91/533/EWG. 
Großbritannien und Irland traditionell eher gering ausgebaut ist (Hepple/Fredman 1992; Prondzynski/Richards 1994). Entsprechend ist zu erwarten, dass die letztgenannten Länder bei der Umsetzung der sechs Richtlinien tendenziell mit einem hohen Maß an erforderlichen Anpassungen konfrontiert sein werden, während für Deutschland und die Niederlande mit eher geringem Anpassungsbedarf zu rechnen ist.

Da das Konzept des Anpassungsbedarfs in der Literatur nicht immer einheitlich gebraucht wird, habe ich eine breit angelegte Konzeptualisierung gewählt, um möglichst viele der vertretenen Auffassungen abdecken zu können. Ich unterscheide vier Dimensionen von Anpassungen, die von einer europäischen Richtlinie und ihrer Umsetzung hervorgerufen werden können. Erstens geht es um die materielle Regelungsebene, d.h. um graduelle oder qualitative Veränderungen an den bestehenden nationalen Politiktraditionen, wobei hier sowohl die rechtliche Ebene als auch die Implikationen der rechtlichen Anpassungen in der Praxis von Bedeutung sind. Zweitens können auch Reformen der administrativen Strukturen erforderlich werden, etwa die Einrichtung einer neuen Überwachungsbehörde. Drittens kann die Umsetzung einer europäischen Vorschrift Veränderungen in den Staat-Verbände-Beziehungen erfordern, indem beispielsweise allgemeinverbindliche gesetzliche Regelungen in einem Bereich eingeführt werden müssen, der zuvor der autonomen Regelung durch Tarifverträge unterlag. Viertens ist schließlich auf die ökonomischen Kosten der übrigen drei Formen des Anpassungsbedarfs zu achten, d.h. auf die finanziellen Implikationen für den Staatshaushalt und für die Privatwirtschaft bzw. für einzelne Sektoren. Ähnlich wie bereits von Knill und Lenschow (1998) vorgeschlagen, nehme ich eine dreigeteilte Kategorisierung der jeweiligen Anpassungserfordernisse in die Stufen "hoch“, „mittel“ und „gering" vor. Daneben gibt es natürlich auch noch den Fall, dass überhaupt keine entsprechenden Anpassungen erforderlich sind.

Insgesamt zeigte sich bei der empirischen Ermittlung des jeweiligen Anpassungsbedarfs in den einzelnen Fällen, dass die indirekten Formen des Anpassungsbedarfs, also Veränderungen der administrativen Strukturen und der Staat-Verbände-Beziehungen, in den hier untersuchten Fällen keine Rolle spielten. Die notwendigen Reformen konzentrierten sich auf die materielle Regelungsebene und die dadurch verursachten Kosten. Es sind allerdings sicherlich andere Fälle denkbar, in denen auch diese Dimensionen von Bedeutung sind. ${ }^{3}$

Konfrontiert man die Werte für den jeweils erzeugten „misfit“ der Richtlinien in den vier Ländern mit dem beobachteten Anpassungserfolg, d.h. mit der Pünktlichkeit und inhaltlichen Korrektheit der Umsetzung, so ergibt sich ein gemischtes Bild, das den Erwartungen der Misfit-These nur sehr begrenzt entspricht (siehe Tabelle 1).

Rund ein Drittel der Fälle weist ein Muster auf, das den Erwartungen der These widerspricht - zumeist in Form von erheblichen Verzögerungen oder mangelhafter Umsetzung trotz geringer Anpassungserfordernisse. Weitere sieben der untersuchten Umsetzungsprozesse liegen im Bereich mittleren Anpassungsbedarfs, für den die misfitorientierte Sichtweise keine eindeutigen Aussagen trifft. Knill und Lenschow (2001: 124 und 126) verweisen zur Erklärung solcher Fälle summarisch auf eine „supportive

3 Im Rahmen des eingangs erwähnten MPIfG-Projektverbundes wurden auch die übrigen elf Mitgliedsstaaten untersucht. Dabei zeigte sich, dass manche der hier analysierten Richtlinien, etwa in Dänemark, Anpassungsbedarf im Bereich der Staat-Verbände-Beziehungen erzeugten. 
Tabelle 1: Anpassungsbedarf, Anpassungserfolg und die Misfit-These

\begin{tabular}{|c|c|c|c|c|}
\hline \multirow{2}{*}{\multicolumn{2}{|c|}{$\begin{array}{l}\text { Zeitpunkt und inhaltliche } \\
\text { Richtlinienkonformität der } \\
\text { Umsetzung }\end{array}$}} & \multicolumn{3}{|c|}{ Anpassungsbedarf } \\
\hline & & hoch & mittel & gering/null \\
\hline \multirow[t]{2}{*}{$\begin{array}{l}\text { weit gehend } \\
\text { rechtzeitig }\end{array}$} & $\begin{array}{l}\text { weit gehend } \\
\text { korrekt }\end{array}$ & GB Mutterschutz & $\begin{array}{l}\text { NL Elternurlaub } \\
\text { GB Teilzeit } \\
\text { IRL Mutterschutz }\end{array}$ & $\begin{array}{l}\text { (D Teilzeit) } \\
\text { NL Nachweis } \\
\text { NL Mutterschutz } \\
\text { NL Arbeitszeit } \\
\text { NL Teilzeit } \\
\text { GB Nachweis }\end{array}$ \\
\hline & $\begin{array}{l}\text { in wichtigen } \\
\text { Teilen inkorrekt }\end{array}$ & $\begin{array}{l}\text { (GB Elternurlaub) } \\
\text { (IRL Elternurlaub) }\end{array}$ & & D Arbeitszeit \\
\hline \multirow{2}{*}{$\begin{array}{l}6-12 \text { Monate } \\
\text { verspätet }\end{array}$} & $\begin{array}{l}\text { weit gehend } \\
\text { korrekt }\end{array}$ & & IRL Teilzeit & $\begin{array}{l}\text { D Jugendliche } \\
\text { IRL Nachweis }\end{array}$ \\
\hline & $\begin{array}{l}\text { in wichtigen } \\
\text { Teilen inkorrekt }\end{array}$ & & & \\
\hline \multirow{2}{*}{$\begin{array}{l}12-24 \\
\text { Monate } \\
\text { verspätet }\end{array}$} & $\begin{array}{l}\text { weit gehend } \\
\text { korrekt }\end{array}$ & (IRL Arbeitszeit) & & D Nachweis \\
\hline & $\begin{array}{l}\text { in wichtigen } \\
\text { Teilen inkorrekt }\end{array}$ & (GB Arbeitszeit) & & NL Jugendliche \\
\hline \multirow{2}{*}{$\begin{array}{l}\text { mehr als } 24 \\
\text { Monate } \\
\text { verspätet }\end{array}$} & $\begin{array}{l}\text { weit gehend } \\
\text { korrekt }\end{array}$ & & $\begin{array}{l}\text { GB Jugendliche } \mathrm{II}^{\mathrm{a}} \\
\text { IRL Jugendliche }\end{array}$ & $\begin{array}{l}\text { D Elternurlaub } \\
\text { GB Jugendliche } \mathrm{I}^{\mathrm{a}}\end{array}$ \\
\hline & $\begin{array}{l}\text { in wichtigen } \\
\text { Teilen inkorrekt }\end{array}$ & & D Mutterschutz & \\
\hline
\end{tabular}

Dunkel unterlegte Felder enthalten Fälle, die der Misfit-These widersprechen. Weiße Felder kennzeichnen empirische Bereiche, für welche die These keine eindeutige Aussage trifft. Hell unterlegte Felder kennzeichnen Fälle, die grundsätzlich mit der Misfit-These vereinbar sind. Fälle in Klammern entsprechen den Erwartungen der These nur scheinbar, sind aber bei einer genaueren Analyse auf andere Wirkungsmechanismen zurückzuführen.

a Die Jugendarbeitsschutz-Richtlinie gewährte Großbritannien eine sechsjährige Übergangsfrist für die Umsetzung einiger ihrer wesentlichen Bestimmungen. Daher wird dieser Umsetzungsprozess hier durch zwei getrennte Fälle wiedergegeben.

actor constellation", die aber nicht mehr weiter systematisch ausdifferenziert wird. Autoren wie Börzel (2000a, 2000b) und Duina $(1997,1999)$ decken diesen empirisch durchaus nicht unbedeutend erscheinenden Bereich mittlerer Anpassungserfordernisse mit ihren Konzeptualisierungen erst gar nicht ab. Deutlich weniger als die Hälfte aller Fälle entspricht auf den ersten Blick den Erwartungen der Hypothese, wobei eine detaillierte empirische Analyse ergibt, dass in fünf dieser scheinbar passenden Fälle andere Erklärungsfaktoren neben der Höhe des Anpassungsbedarfs eine entscheidende Rolle gespielt haben. Ich werde im nächsten Abschnitt etwas genauer auf einige dieser Fälle eingehen, um dies zu verdeutlichen. Somit verbleiben lediglich fünf von 25 Fällen, bei denen die beobachtete Art der Umsetzung allein unter Rückgriff auf die Höhe des Anpassungsbedarfs erklärt werden kann. 


\section{Parties do matter: europäische Anpassungserfordernisse und die parteipolitische Logik ihrer Verarbeitung}

Die Ergebnisse meiner Fallstudien machen deutlich, dass bei der Umsetzung die Eigenlogik nationaler politischer Prozesse wesentlich genauer in den Blick genommen werden muss als dies von den Vertretern einer auf strukturelle Inkompatibilitäten ausgerichteten Sichtweise bislang getan wurde. Ich gehe daher von einem akteurzentrierten Ansatz aus, der nationalen Regierungen, Verwaltungen und Interessengruppen ein eigenes Gewicht bei der Umsetzung einräumt. Insbesondere argumentiere ich, dass die Haltungen dieser Akteure im Rahmen der Umsetzung nicht durch den zu überwindenden „misfit“ determiniert sind. Nationale Akteure haben vielmehr eigene Präferenzen, auf deren Grundlage sie die von europäischen Richtlinien geforderten Anpassungen beurteilen.

In meinen empirischen Analysen über die Umsetzung arbeitsrechtlicher Richtlinien hat sich in diesem Zusammenhang der Einfluss von politischen Parteien als besonders wichtig erwiesen. Die unterschiedliche parteipolitische Ausrichtung der Regierung spielte in rund der Hälfte der analysierten Fälle eine entscheidende Rolle. Daher konzentriere ich mich hier auf die Wirkungsweise dieses Faktors. Mein Argument lautet, dass die Haltungen nationaler Regierungen gegenüber der Umsetzung europäischer Richtlinien nur zu einem geringen Teil von der Höhe des Anpassungsbedarfs und den daraus resultierenden Kosten bestimmt sind. Regierungen agieren nicht als reine Verteidiger des nationalen Status quo. Sehr viel bedeutender erwiesen sich hingegen die parteipolitisch definierten Präferenzen der Regierungen. Wenn diese aktiviert werden, so sind zwei Reaktionsmuster mit jeweils unterschiedlichen Implikationen für die Rechtzeitigkeit und inhaltliche Qualität der Umsetzung zu unterscheiden:

- Widerspricht eine Richtlinie den parteipolitisch definierten Zielen der Regierung, so ist wahrscheinlich, dass diese unabhängig vom Anpassungsbedarf eine explizit ablehnende Haltung einnimmt. In diesem Fall kommt es mit hoher Wahrscheinlichkeit zu Verzögerungen oder absichtsvoller Falschumsetzung.

- Harmonieren die geforderten Anpassungen jedoch mit den parteipolitisch determinierten Präferenzen der Regierung, so ist selbst bei hohem Anpassungsbedarf mit $a k$ tiver Unterstützung zu rechnen, die sich häufig in Form von schneller und korrekter Umsetzung oder auch in Gestalt von Überimplementation bemerkbar macht.

Im Folgenden werde ich diese Reaktionsmuster anhand empirischer Beispiele verdeutlichen, bevor ich mich dann der Frage zuwende, welche Parteikonstellationen welche Reaktionen wahrscheinlich machen und inwiefern meine Befunde mit den Erwartungen der Parteiendifferenz-These in Einklang stehen.

\subsection{Parteipolitischer Widerstand, Verschleppung und Umsetzungsmängel}

Parteipolitisch begründeter Widerstand gegen eine Richtlinie und ihre Umsetzung kann sich innerhalb der Regierung an verschiedenen Stellen manifestieren. Dabei sind zunächst Alleinregierungen und Koalitionsregierungen zu unterscheiden. Bei Koalitionen kommt es wiederum darauf an, ob die betreffende Partei das primär zuständige Ressort innehat oder lediglich ein anderes Ministerium besetzt. Im Fall von allein re- 
gierenden Parteien und von Parteien, die das zuständige Ressort innehaben, führt explizite Opposition gegen bestimmte Aspekte einer Richtlinie häufig zum bewussten „Versanden-Lassen“ der Umsetzung, während Widerstand auf Seiten einer anderen Koalitionspartei Konflikte zwischen den Parteien innerhalb der Regierungskoalition nach sich zieht, wodurch es zu beträchtlichen Verzögerungen kommen kann. Parteipolitischer Widerstand kann darüber hinaus auch zu absichtsvoller Falschumsetzung führen.

\subsubsection{Parteipolitischer Widerstand und bewusstes „Versanden-Lassen“ der Umsetzung}

Wenn die Partei, deren Zielen eine bestimmte europäische Vorschrift widerspricht, allein regiert, so ist es für sie in der Regel sehr einfach, ihren Widerwillen in die Tat umzusetzen. Diese Konstellation war unter den untersuchten Ländern lediglich in Großbritannien gegeben. In diesem Fall verwandelte sich die geringe Zahl parteipolitischer Vetopunkte von einem Instrument effektiver Politikgestaltung in ein ebenso effektives Blockadeinstrument.

Das zeigte sich besonders deutlich bei der britischen Umsetzung der ArbeitszeitRichtlinie. Dort sträubte sich die Tory-Regierung unter John Major bis zu ihrer Abwahl mit allen Mitteln dagegen, die europäischen Bestimmungen zu übernehmen. Auf den ersten Blick stimmt die Opposition der britischen Regierung mit den Erwartungen der Misfit-These überein, denn die Umsetzung der Vorschriften bedeutete für Großbritannien signifikanten Anpassungsbedarf und immense Kosten. So gab es in Großbritannien zuvor weder einen gesetzlichen Anspruch auf bezahlten Jahresurlaub noch irgendwelche gesetzlichen Vorschriften über Arbeits- und Ruhezeiten. Dieser Mangel an staatlich definierten Rahmenbedingungen für die Arbeitszeitgestaltung der Unternehmen war gepaart mit einer ausgesprochenen „long-hours culture“ (Interview GB 4: 187). Nach Schätzungen der Regierung arbeiteten Anfang der 1990er Jahre rund 2,7 Millionen Arbeitnehmer (fast 12 Prozent der Gesamtbeschäftigten) regelmäßig mehr als die von der Richtlinie vorgegebene Höchstgrenze von 48 Stunden pro Woche (FT, 30.4.1992: 9).

Ihre eigentliche Brisanz erhielt die Richtlinie jedoch erst dadurch, dass der Vorschlag der Kommission auf eine spezifische Mischung aus euroskeptischen und antiinterventionistischen Grundüberzeugungen innerhalb der konservativen Regierungspartei traf. Vor diesem Hintergrund erschien die Richtlinie nicht nur als Maßnahme, welche die Kosten der britischen Wirtschaft in die Höhe trieb, sondern als wirtschaftspolitisch falsche und illegitime Einmischung der Brüsseler Bürokratie in die inneren Angelegenheiten Großbritanniens. ${ }^{4}$ Da die Kommission die Arbeitszeit-Richtlinie im Rah-

4 So argumentierte Premierminister Major in einer Rede vor der Jahresversammlung des Institute of Directors im April 1992, er sei keinesfalls bereit, „to let Brussels intervene in areas which Westminster had decided to leave alone." Konkret bezeichnete er die Arbeitszeit-Vorschläge als „unnecessary interference with working practices“ und fügte hinzu: „They are not for us. No one should be in any doubt. A Conservative government will strongly oppose such damaging regulation wherever it is found, and we will not readily acquiesce in any attempts to impose these costs on our industry" (zitiert in FT, 29.4.1992: 14). 
men ihres „treaty-base game“ (Rhodes 1995: 99) als Sicherheits- und Gesundheitsschutz-Maßnahme auf Grundlage von Artikel 118a EGV eingebracht hatte, reichte zur Verabschiedung eine qualifizierte Mehrheit im Rat. Die britische Regierung protestierte zwar gegen die Wahl dieser Rechtsgrundlage und argumentierte, dass es sich bei den vorgeschlagenen Maßnahmen um Arbeitsmarktregulierungen handle, die daher der Einstimmigkeit bedürften (Gray 1998: 327-329). Sie konnte allerdings nicht verhindern, dass die Richtlinie schließlich im November 1993 auch gegen erbitterten britischen Widerstand verabschiedet wurde.

Anstatt ihre Niederlage zu akzeptieren und die Richtlinie umzusetzen, strengte die Regierung Major jedoch umgehend eine Klage vor dem Europäischen Gerichtshof wegen der vermeintlich falschen Rechtsgrundlage der Richtlinie an und ließ ebenfalls verlauten, dass sie bis zur Urteilsfindung keinerlei Maßnahmen zur Umsetzung unternehmen werde (FT, 2.6.1993: 1). In seinem Urteil vom 12. November 1996 wies der EuGH die Argumente der britischen Regierung allerdings zurück und bestätigte damit die Rechtskräftigkeit der Richtlinie. ${ }^{5}$ Doch auch jetzt war die konservative Regierung nicht bereit, den Richterspruch zu akzeptieren und die Richtlinie nun umgehend umzusetzen. Vielmehr bekräftigte sie noch am Tag der Urteilsverkündung ihre grundsätzliche Ablehnung des Urteils und verlangte eine entsprechende Revision des Vertragstextes in der laufenden Regierungskonferenz, um auf diesem Wege die Richtlinie für Großbritannien außer Kraft zu setzen (House of Commons Hansard Debates, 12.11. 1996, Col. 152 und 155). Zwar legte die Regierung im Dezember 1996 immerhin ein erstes Konsultationsdokument vor. Bis zu den Unterhauswahlen im Mai 1997, bei denen die Konservativen eine verheerende Niederlage gegen Labour einstecken mussten, wurden dann jedoch keinerlei Schritte mehr unternommen, um die aus der Richtlinie resultierenden europarechtlichen Verpflichtungen zu erfüllen.

Erst die nachfolgende Labour-Regierung unter Tony Blair setzte die Richtlinie schließlich um. Die Labour Party war unter anderem mit dem Versprechen in den Wahlkampf gegangen, sie werde die Arbeitszeit-Richtlinie umsetzen, wenn sie gewählt würde (Interview GB 3: 532-535). Dieses Versprechen setzte die Labour-Regierung dann auch relativ zügig in die Tat um. Aufgrund der bewussten Verzögerungstaktik ihrer konservativen Vorgängerin war dies allerdings erst rund zwei Jahre nach Ablauf der Umsetzungsfrist möglich. ${ }^{6}$ Die unterschiedlichen Haltungen der konservativen Regierung und ihrer Labour-Nachfolgerin gegenüber der Richtlinie machen deutlich, dass die signifikante Verzögerung bei der Umsetzung nicht mit dem hohen Anpassungsbe-

5 Urteil des Gerichthofs vom 12. November 1996, Vereinigtes Königreich gegen Rat der Europäischen Union, Rs. C-84/94, Sammlung der Rechtsprechung 1996, S. I-5755.

6 Das britische Gesetz erwies sich später in einem wesentlichen Punkt als unzureichend und musste schließlich im Oktober 2001 nachgebessert werden. Die britische Mediengewerkschaft BECTU hatte eine Klage angestrengt, da die bestehende britische Urlaubsregelung eine Mindestbeschäftigungsdauer von 13 Wochen voraussetzte. Dadurch wurden Arbeitnehmer mit befristeten Verträgen von sehr kurzer Dauer, wie sie in der britischen Medienbranche weit verbreitet waren, komplett vom Anspruch auf Jahresurlaub ausgeschlossen. Der Richtlinientext war in diesem Punkt nicht eindeutig. Die BECTU-Klage wurde daher an den EuGH verwiesen, und dieser entschied schließlich, dass eine solche Mindestbeschäftigungszeit gegen die Richtlinie verstoße. Siehe Urteil des Gerichtshofes vom 26. Juni 2001, The Queen gegen Secretary of State for Trade and Industry, Rs. C-173/99, Sammlung der Rechtsprechung 2001, S. I-4881. 
darf zu erklären ist. Entscheidend waren die unterschiedlichen parteipolitischen „Brillen" beider Regierungen, die bei der einen zu Fundamentalopposition und bei der anderen zu Unterstützung oder doch zumindest zu Akzeptanz der Brüsseler Vorschriften führte.

Derselbe Effekt der absichtsvollen Nichtumsetzung aufgrund politisch motivierter Opposition kann unter Koalitionsregierungen auftreten, wenn die anpassungsunwillige Partei die Leitung des federführenden Ministeriums innehat und innerhalb der Regierungskoalition keine anderen Akteure existieren, die ein Interesse an der Umsetzung hätten. Dies lässt sich anhand der Umsetzung der Elternurlaubs-Richtlinie in Deutschland verdeutlichen. Insgesamt war der Anpassungsbedarf gering, da das deutsche Bundeserziehungsgeldgesetz bereits eine vergleichsweise großzügige gesetzliche Erziehungsurlaubs-Regelung vorsah, die teilweise weit über die Mindeststandards der Richtlinie hinausging.

Lediglich ein insgesamt geringfügig erscheinender Punkt musste aufgrund der Richtlinie geändert werden: Während die Richtlinie ein individuelles Recht auf Elternurlaub für Frauen und Männer vorsah, war der Anspruch auf Erziehungsurlaub in Deutschland vom Beschäftigungsstatus des jeweils anderen Elternteils abhängig. So hatte ein Arbeitnehmer keinen Anspruch auf Erziehungsurlaub, sofern „der mit dem Arbeitnehmer in einem Haushalt lebende andere Elternteil nicht erwerbstätig ist" ( $\$ 15$ des BErzGG i. d. Fassung v. 31.1.1994; siehe auch Hornung-Draus 1996). Typischerweise betraf diese Regelung Paare, bei denen der Mann erwerbstätig war, während die Frau als Hausfrau zu Hause arbeitete. Die Zahl solcher Paare ist zwar zahlenmäßig nicht zu vernachlässigen, ${ }^{7}$ faktisch sind von der Erweiterung des Elternurlaubsanspruchs auf diese Eltern allerdings nur sehr geringe Auswirkungen zu erwarten. Denn angesichts der insgesamt geringen Höhe des Erziehungsgeldes ist es eher unrealistisch, dass Paare, die allein auf das Einkommen des einen erwerbstätigen Elternteils angewiesen sind, gerade auf dieses Einkommen verzichten und stattdessen versuchen, ausschließlich vom Erziehungsgeld zu leben.

Trotz der geringen praktischen Bedeutung der erforderlichen Reform, die sich auch darin ausdrückte, dass weder Gewerkschaften noch Arbeitgeberverbände größeres Interesse dafür zeigten (Interviews D 5, D 9), weigerte sich die konservativ-liberale Regierung unter Helmut Kohl, die von ihr selbst Mitte der 1980er Jahre geschaffene Regelung zu ändern. Das lag daran, dass die erforderliche Anpassung der familienpolitischen Konzeption insbesondere des konservativen Koalitionspartners widersprach. Bereits bei der Verabschiedung der Richtlinie gab die Bundesregierung zu Protokoll, dass

7 Nach Angaben des Statistischen Bundesamtes lag die Erwerbstätigenquote verheirateter Frauen im Alter zwischen 15 und 65 Jahren im Jahr 2001 bei knapp unter 60 Prozent (Statistisches Bundesamt 2002: 60). Demnach wären etwa 40 Prozent aller Ehepaare als potenziell betroffene Alleinverdienerehepaare zu klassifizieren. Diese Zahl überschätzt aber den tatsächlichen Umfang der Nutznießer, da sie auch Frauen erfasst, die keine Kinder mehr bekommen können oder dies gar nicht wollen. Richtet man den Blick stärker auf jüngere Frauen, wie es eine im Jahr 2000 durchgeführte Umfrage des Instituts für Arbeitsmarkt- und Berufsforschung tut, so ergibt sich ein wesentlich geringerer Anteil weiblicher Nichterwerbstätiger. Nach dieser Untersuchung gingen nur rund 14 Prozent aller befragten Frauen keiner Erwerbstätigkeit nach (eigene Berechnungen auf Basis der Daten in Beckmann/Kurtz 2001). Mit anderen Worten: Etwa jedes siebte Paar hätte grundsätzlich von der Neuregelung profitieren können. 
aus ihrer Sicht „eine Änderung des deutschen Rechts nicht erforderlich und nicht beabsichtigt“ sei, da die Richtlinie den Mitgliedsstaaten „eine große Flexibilität hinsichtlich der Bedingungen für die Gewährung und die Inanspruchnahme des Elternurlaubs“ einräume (Ministerrat 1996: 6). Weil das zuständige Familienministerium von der CDU-Politikerin Claudia Nolte geleitet wurde und es sonst niemanden innerhalb der Regierung gab, der sich für die nötigen Anpassungen aussprach, war es ein Leichtes, die Umsetzung stillschweigend „versanden“ zu lassen.

Erst unter der 1998 an die Macht gekommenen rot-grünen Bundesregierung wurde dann eine umfassende Reform der Erziehungsurlaubs-Vorschriften durchgeführt. Die am 1. Januar 2001 in Kraft getretene Reform verwirklichte nicht nur, wenn auch mit erheblicher Verspätung, die von der Richtlinie geforderte Ausdehnung des Elternurlaub-Anspruchs auf Paare mit nur einem Einkommen, sondern griff gleichzeitig auch noch eine Reihe unverbindlicher Empfehlungen der Richtlinie auf und ging auf diese Weise zum Teil weit über die europäischen Mindestanforderungen hinaus. So wurde die Teilzeitarbeitsmöglichkeit während des Erziehungsurlaubs von 19 auf 30 Wochenstunden erhöht und Arbeitnehmern ein verbindlicher Rechtsanspruch auf Teilzeitarbeit während des Elternurlaubs gewährt. Es zeigt sich also, dass auch geringfügige Anpassungen zum Scheitern verurteilt sind, wenn sie den politischen Präferenzen wichtiger Regierungsparteien zuwiderlaufen, während sie von einer Regierung, deren ideologisches Profil mit den vorgegeben Zielen übereinstimmen, problemlos akzeptiert und durch Überimplementation sogar noch „vergoldet“ werden können.

\subsubsection{Parteipolitischer Widerstand, parteipolitische Vetospieler und verzögerte Umsetzung}

Parteipolitischer Widerstand kann auch dazu führen, dass innerhalb einer Regierungskoalition ein oder mehrere Koalitionspartner gegen die vorgeschlagene Art der Umsetzung opponieren und es darüber zu einem koalitionsinternen Streit kommt. Da innerhalb einer Regierungskoalition alle beteiligten Parteien über Vetomacht verfügen (Tsebelis 1995), kann auf diese Weise ein langwieriger und schwer zu lösender regierungsinterner Konflikt entstehen. Das zeigte sich bei der Umsetzung der Nachweis-Richtlinie in Deutschland. Die Richtlinie bedeutete für Deutschland nur geringen Anpassungsbedarf, da es zwar keine gesetzliche Regelung gab, die den Arbeitnehmern das Recht auf einen schriftlichen Nachweis über die wesentlichen Bedingungen ihres Arbeitsverhältnisses zusicherte, wohl aber Tarifverträge, die entsprechende Rechte garantierten, zumeist in Form des Anspruchs auf einen schriftlichen Arbeitsvertrag. Auf diese Weise waren mehr als 80 Prozent aller Arbeitnehmer bereits faktisch mit Ansprüchen ausgestattet, die den Anforderungen der Richtlinie weit gehend entsprachen (BR-Drucksache 353/94: 13-14).

Trotz der insgesamt geringfügigen Auswirkungen für den Großteil der Unternehmen kämpfte das FDP-geführte Wirtschaftsministerium für eine möglichst weit gehende Vermeidung ,unnötiger Bürokratie“ vor allem für kleinere Betriebe und setzte sich daher vehement für die Nutzung einer Ausnahmeklausel ein, die es erlaubt hätte, Teilzeitarbeitnehmer mit einer wöchentlichen Arbeitszeit von bis zu acht Stunden vom Anwendungsbereich der Regelung auszunehmen. Das Arbeitsministerium unter Norbert 
Blüm, einem der Exponenten des CDU-Arbeitnehmerflügels, argumentierte hingegen, dass ein solcher Ausschluss von Teilzeitarbeitnehmern, obwohl in der Nachweisrichtlinie ausdrücklich erlaubt, eine indirekte Diskriminierung von Frauen darstellen und daher gegen die einschlägige Rechtsprechung des EuGH verstoßen würde. Daher handle es sich hierbei nur um eine "Scheinausnahme“, die nicht genutzt werden könne (Interview D 6: 185-210). Bis dieser langwierige Ressortkonflikt, der aufgrund der unterschiedlichen parteipolitischen Besetzung beider Ministerien vor allem als Koalitionskonflikt zwischen FDP und CDU angesehen werden muss, mit einem Kompromiss gelöst werden konnte, war die Umsetzungsfrist schon lange abgelaufen. ${ }^{8}$

\subsubsection{Parteipolitischer Widerstand und absichtliche Falschumsetzung}

Insgesamt können sich parteipolitisch motivierte Auseinandersetzungen und Widerstände natürlich nicht nur auf der Zeitachse bemerkbar machen, sondern auch in der inhaltlichen Dimension, in Form von absichtlicher Falschumsetzung. Das wurde besonders deutlich bei der Umsetzung der Arbeitszeit-Richtlinie in Deutschland. Die Vorschriften der Richtlinie bedeuteten für die Bundesrepublik nur geringen Anpassungsbedarf, da das existierende deutsche Recht, bestehend aus der Arbeitszeitordnung von 1938 und dem Bundesurlaubsgesetz von 1963, bereits den Großteil der Standards erfüllte und in wichtigen Punkten sogar wesentlich strengere Vorschriften vorsah. Die Richtlinie erforderte im Wesentlichen eine gewisse Verbesserung des Gesundheitsschutzes von Nachtarbeitnehmern (Anzinger 1994: 7) und eine Anhebung des gesetzlichen Mindesturlaubs von drei auf vier Wochen. Die letztere Reform betraf aber faktisch nur einen Bruchteil der Arbeitnehmer, da die überwiegende Mehrzahl der Beschäftigten durch Tarifverträge bereits wesentlich großzügigere Urlaubsansprüche genoss (BTDrucksache 12/6990: 45).

Diese Reformerfordernisse spielten jedoch bei der Umsetzung kaum eine Rolle. Entscheidend für ein Verständnis der deutschen Reaktion auf die Richtlinie ist vielmehr, dass die europäischen Vorschriften mit einer bereits vorher begonnenen, grundlegenden Reform des bestehenden Arbeitszeitrechts in Deutschland kollidierten. Bereits in den 1980er Jahren hatte es mehrere gescheiterte Anläufe der konservativ-liberalen Bundesregierung gegeben, die relativ starren und darüber hinaus mit nationalsozialistischem Jargon durchsetzten Vorschriften der Arbeitszeitordnung grundlegend zu reformieren und vor allem deutlich zu flexibilisieren (siehe dazu Zohlnhöfer 2001: 129136).

1992 wurde dann ein erneuter Anlauf unternommen, der sich mit den ArbeitszeitVerhandlungen auf europäischer Ebene überlagerte. Im Rahmen der geplanten natio-

8 Man einigte sich schließlich darauf, die 8-Stunden-Schwelle zwar nicht zu nutzen, dafür aber eine neue Geringfügigkeitsschwelle einzuführen, die alle Arbeitnehmer vom Anwendungsbereich des Gesetzes ausschloss, deren jährliche Arbeitszeit geringer als 400 Stunden war $(\$ 1$ des Nachweisgesetzes i. d. Fassung v. 20.7.1995). Diese Ausnahme stand im Einklang mit einer anderen Ausnahmeklausel der Nachweis-Richtlinie, die einen Ausschluss von Arbeitnehmern zuließ, die „Gelegenheitsarbeit" verrichten. Inzwischen ist die 400-Stunden-Schwelle von der rot-grünen Bundesregierung abgeschafft worden (Artikel 7 des Gesetzes zur Neuregelung der geringfügigen Beschäftigungsverhältnisse vom 24.3.1999). 
nalen Reform bestand der wichtigste Hebel zur Flexibilisierung neben einer höchst umstrittenen Aufweichung des Sonntagsarbeitsverbots vor allem in einer erheblichen Ausdehnung der Referenzperiode für die Berechnung der durchschnittlichen wöchentlichen Höchstarbeitszeit. Während die Arbeitszeitordnung diesbezüglich nur minimale Flexibilität erlaubte, drängte vor allem das FDP-geführte Wirtschaftsministerium unter Günter Rexrodt auf möglichst lange Referenzperioden, die es den Unternehmen ermöglichen würden, in Stoßzeiten weit über die normalerweise erlaubte wöchentliche Höchstarbeitszeit von 48 Stunden hinauszugehen, sofern diese Arbeitszeitspitzen innerhalb eines bestimmten Zeitraums wieder ausgeglichen würden (FAZ, 15.6.1993: 15; 13.7.1993: 11).

Vor dem Hintergrund dieser nationalen Pläne drängte die Bundesregierung im Rahmen der europäischen Verhandlungen auf die Festlegung möglichst langer Referenzperioden von bis zu 12 Monaten, was allerdings auf den vehementen Widerstand insbesondere der französischen Regierung stieß (ArbuR 1993; Lewis 1998: 388-390). Letztlich konnte sich die deutsche Regierung bei den Verhandlungen mit ihrem Anliegen nicht durchsetzen. Die schließlich verabschiedete Richtlinie sah eine grundsätzliche Referenzperiode von lediglich vier Monaten vor. Der einzige deutsche Erfolg war, dass dieser Bezugszeitraum in bestimmten Sektoren auf sechs Monate und per Tarifvertrag auch auf 12 Monate verlängert werden konnte. Die Niederlage in Brüssel brachte die deutsche Regierung in die unangenehme Lage, den bislang in ihrem Gesetzentwurf vorgesehenen Ausgleichszeitraum von sechs Monaten auf vier Monate reduzieren zu müssen. Obwohl dieser Umstand im Arbeitsministerium durchaus bekannt war (Günther 1993: 20) und für eine entsprechende Anpassung in laufenden Gesetzgebungsverfahren noch genügend Zeit gewesen wäre, hielt die konservativ-liberale Koalition an den vorgesehenen sechs Monaten Ausgleichszeitraum fest. Darüber hinaus erlaubte das letztlich verabschiedete Arbeitszeitgesetz den Sozialpartnern, per Tarifvertrag oder Betriebsvereinbarung längere Ausgleichszeiträume ohne eine gesetzlich definierte Obergrenze zu vereinbaren, während die Richtlinie dafür eine Maximalgrenze von $12 \mathrm{Mo-}$ naten definierte.

Die von vielen Kommentatoren bei ihrer Verabschiedung konstatierte extrem hohe Flexibilität der Richtlinie (siehe etwa European Industrial Relations Review 235, August 1993: 15-18; Lörcher 1994) reichte der Kohl-Regierung für ihre eigenen nationalen Reformen also offenbar nicht aus. Vielmehr wollte sie ihre weit gehenden Flexibilisierungsbestrebungen nicht durch striktere europäische Vorschriften einengen lassen und überschritt daher bei ihrer Reform den vorgegebenen Rahmen der Richtlinie deutlich. Das Resultat dieser parteipolitisch motivierten Entscheidung ist, dass das deutsche Arbeitszeitgesetz seit mehr als sechs Jahren gegen gültiges europäisches Recht verstößt. Darauf hat inzwischen auch die Europäische Kommission hingewiesen, ohne allerdings bislang entsprechende rechtliche Schritte eingeleitet zu haben (KOM[2000] 787: 18).

\subsection{Parteipolitische Unterstützung, effektive Umsetzung und Tendenz zur Überimplementation}

Im Gegensatz zu den bisher beschriebenen Beispielen parteipolitischen Widerstands trug die parteipolitisch motivierte Unterstützung einer Richtlinie durch die zuständige 
Regierung in einigen Fällen zu einer schnellen und konformen Umsetzung bei. Darüber hinaus haben meine empirischen Analysen gezeigt, dass parteipolitische Unterstützung auch dazu führen kann, dass im Rahmen der Umsetzung über die Mindestanforderungen der Richtlinie hinausgegangen wird.

In mehreren der untersuchten Fälle trug die parteipolitisch begründete aktive Unterstützung einer Richtlinie wesentlich zur schnellen und konformen Anpassung bei. Die bereits erwähnten Beispiele von Umsetzungsverschleppung durch unwillige MitteRechts-Regierungen in Deutschland (Elternurlaub) und Großbritannien (Arbeitszeit) konnten nach einem entsprechenden Regierungswechsel hin zu sozialdemokratisch geführten Regierungen relativ schnell überwunden werden.

Parteipolitische Unterstützung kann aber auch zu Überimplementation führen. So griff die rot-grüne Bundesregierung bei der bereits erwähnten Reform der deutschen Erziehungsurlaubs-Regelung einige der unverbindlichen Bestimmungen der Elternurlaubs-Richtlinie auf, weil sie mit den familienpolitischen Konzeptionen der beiden Koalitionspartner SPD und Grüne in Einklang standen. Auf diese Weise ging die Regierung bei der Umsetzung weit über die europäischen Mindestanforderungen hinaus.

Analog dazu lässt sich die massive Überimplementation der Teilzeitarbeit-Richtlinie in Deutschland interpretieren. Das Abkommen der europäischen Sozialpartner über Teilzeitarbeit, auf dem die Richtlinie inhaltlich basierte, war bei seiner Verabschiedung von verschiedenen Beobachtern, unter anderem von den deutschen Gewerkschaften, aufgrund seiner mangelnden Substanz harsch kritisiert worden. ${ }^{9}$ Das einzige verbindliche Prinzip der Richtlinie legt fest, dass Teilzeitarbeitnehmer hinsichtlich ihrer Beschäftigungsbedingungen nicht schlechter behandelt werden dürfen als vergleichbare Vollzeitarbeitnehmer. Dieses Prinzip war in Deutschland bereits weit gehend verwirklicht - die Richtlinie verlangte nur eine graduelle Verbesserung des deutschen Beschäftigungsförderungsgesetzes ( $\$ 2$ des BeschFG vom 26.4.1985, siehe auch Interview D 1: 201-216). Ansonsten besteht die Richtlinie zu weiten Teilen aus unverbindlichen Empfehlungen. Bei der Umsetzung der Richtlinie in Deutschland durch die rot-grüne Bundesregierung wurden jedoch fast alle dieser nicht verpflichtenden Bestimmungen aufgegriffen und in bindende Vorschriften mit teilweise weit reichenden Konsequenzen umgewandelt.

Besonders deutlich wird dies bei der Frage der Förderung von Teilzeitarbeit. Die Richtlinie enthält hierzu lediglich eine sehr weich formulierte Bestimmung, die besagt, dass Arbeitgeber Anträge von Vollzeitbeschäftigten auf Wechsel in ein im Betrieb zur Verfügung stehendes Teilzeitarbeitsverhältnis berücksichtigen sollten, soweit dies möglich ist ( $\$$ 5.3.c des Abkommens). Im deutschen Teilzeit- und Befristungsgesetz wurde daraus ein Rechtsanspruch auf Teilzeitarbeit gemacht, der für alle Arbeitnehmer gilt, die in Betrieben mit mehr als 15 Beschäftigten arbeiten ( $\$ 8$ des TzBfG). Eine Verringerung der wöchentlichen Arbeitszeit kann nunmehr gerichtlich eingeklagt werden, wobei der Arbeitgeber "betriebliche Gründe“ vorbringen muss, um einen solchen Antrag auf Wechsel in ein Teilzeitarbeitsverhältnis ablehnen zu können. Dieses weit reichende Aufgreifen unverbindlicher Bestimmungen lässt sich auch noch in anderen Bereichen

9 Bei der Abstimmung über das Rahmenabkommen innerhalb des EGB stimmten unter anderem die deutschen Gewerkschaften und Teile der französischen und luxemburgischen Verbände gegen das Abkommen, weil es zu wenig Verbesserungen bringen würde (Dürmeier 1999). 
der Richtlinie beobachten. Es ist damit zu erklären, dass die Förderung der Teilzeitarbeit von der Bundesregierung als probates Mittel zur Verringerung der Arbeitslosigkeit angesehen wurde, weswegen durchgreifende gesetzliche Schritte zur Förderung dieser Art von Beschäftigung ergriffen werden sollten (BR-Drucksache 591/00: 12-15).

Um die massiven Proteste des Arbeitgeberlagers gegen diese „unnötige“ Regulierung des Arbeitsmarktes zu beschwichtigen, schnürte die Regierung ein Paket, das die Umsetzung der Teilzeitarbeits-Richtlinie mit der Implementation der Richtlinie über befristete Arbeitsverträge verknüpfte. Die Arbeitgeber hatten an der gesetzlichen Verankerung der Befristungsregelung großes Interesse, denn das Beschäftigungsförderungsgesetz, auf dessen Grundlage der Abschluss befristeter Arbeitsverträge ohne sachlichen Grund erlaubt wurde, wäre Ende 2000 ausgelaufen. Ohne entsprechendes Nachfolgegesetz wäre der Abschluss befristeter Verträge ab Januar 2001 faktisch kaum mehr möglich gewesen. Dieser package deal sicherte die rechtzeitige Verabschiedung der Teilzeit-Regelung, die angesichts des Widerstands der Arbeitgeber sonst eher fraglich gewesen wäre. Doch auch mit diesem Paket musste die Bundesregierung noch von der Möglichkeit einer einjährigen Verlängerung der Umsetzungsfrist Gebrauch machen, die in der Richtlinie vorgesehen war (Interviews D 1: 957-965; D 5: 397-413, 729-779; D 6: 750-783).

\section{3 Überimplementation als Problem rechtzeitiger Anpassung}

Anhand des eben erwähnten Beispiels wird zugleich deutlich, dass parteipolitische Unterstützung nicht nur positive Effekte auf die Umsetzungsqualität von Richtlinien haben muss, sondern auch zu Problemen in der Zeitdimension führen kann, wenn durch inhaltliches „Draufsatteln“ zusätzliche Konflikte entstehen, welche die Umsetzung der verbindlichen Standards verzögern können. Dieser Mechanismus zeigte sich insbesondere bei der Umsetzung der Arbeitszeit-Richtlinie in Irland. Ähnlich wie in Großbritannien bedeutete die Richtlinie für Irland hohen Anpassungsbedarf und signifikante Kosten. In Irland existierten zwar gesetzliche Vorschriften über tägliche und wöchentliche Arbeits- und Ruhezeiten, diese galten jedoch nicht für alle Branchen, sahen zum größten Teil wesentlich weniger strikte Vorschriften vor als die Richtlinie und waren vor allem bereits so alt, dass sie nach allgemeiner Ansicht in der Praxis kaum mehr von Bedeutung waren (Interview IRL 4: 263-267). So arbeiteten nach Schätzung der Regierung 1993 etwa sechs bis sieben Prozent aller Arbeitnehmer in Irland länger als durchschnittlich 48 Stunden pro Woche (Irish Times, 26.8.1993: 15). Darüber hinaus musste der gesetzliche Jahresurlaub von drei auf vier Wochen verlängert werden, was zumindest den rund 20 Prozent der Beschäftigten zugute kam, die noch keine entsprechenden tarifvertraglichen Ansprüche genossen (Dáil Debates, 26.11.1996: 56-57).

Trotz der hohen Anpassungskosten unterstützte die damals amtierende MitteLinks-Regierungskoalition aus Fine Gael, Labour und den Progressive Democrats die Ziele der Richtlinie. Generell versprach sich die Regierung von der erheblichen Reduzierung der erlaubten Wochenarbeitszeit eine Entlastung des Arbeitsmarktes durch Arbeitsumverteilungseffekte. Dieses Ziel wurde insbesondere von der Labour Party unterstützt, die diesbezüglich den Vorteil hatte, dass sie über die Leitung des Arbeitsressorts verfügte. Die zuständige Labour-Staatssekretärin Eithne Fitzgerald schlug daher vor, 
eine Ausnahmeklausel, die es einzelnen Arbeitnehmern erlaubt hätte, durch eine individuelle Opt-out-Erklärung länger als durchschnittlich 48 Stunden pro Woche zu arbeiten, nicht zu nutzen, da eine solche individuelle Opt-out-Möglichkeit die arbeitsmarktpolitischen Effekte der Richtlinie konterkariert hätte (Dáil Debates, 26.11.1996: 53-68, Irish Times, 15.11.1996: 4).

Diese Form der Überimplementation wurde von den Gewerkschaften begrüßt, rief aber vehemente Proteste von Seiten der Arbeitgeberverbände und der Oppositionsparteien hervor, die dadurch vor allem die Wettbewerbsposition Irlands gegenüber den britischen Nachbarn im Kampf um amerikanische Direktinvestitionen gefährdet sahen, da die Briten zu jener Zeit bereits deutlich gemacht hatten, dass sie die Ausnahmeklausel nutzen würden (Dáil Debates, 10.4.1997: 784-785, 792-794, Irish Times, 22.11. 1996: 4, 25.2.1997: 16). Angesichts dieser Proteste vereinbarte die Regierung einen Kompromiss, der eine Nutzung der Opt-out-Möglichkeit unter eng begrenzten Bedingungen und nur für eine Übergangsfrist von zwei Jahren erlaubte. Damit hielt sie im Wesentlichen an ihren Plänen fest. Allerdings führten die Debatten über die Nutzung der Opt-out-Klausel und die Suche nach einem Kompromiss zu einer Verzögerung bei der Umsetzung, die bei einer strikten Minimalinterpretation der Richtlinie zumindest hätte vermindert werden können.

\subsection{Die Umsetzung arbeitsrechtlicher EU-Richtlinien und die Parteiendifferenz-These}

Bei den hier vorgestellten empirischen Fällen kam es zu expliziter Ablehnung arbeitsrechtlicher Richtlinien tendenziell eher bei Mitte-Rechts-Regierungen, während eine aktive Unterstützung solcher Reformen vor allem bei Mitte-Links-Regierungen zu beobachten war. Dieser Befund scheint zunächst die Erwartungen der in der national vergleichenden Politikforschung schon lange diskutierten Parteiendifferenz-These oder „Parties-domatter"-These zu bestätigen, wonach die unterschiedliche parteipolitische Zusammensetzung von Regierungen, und insbesondere die Verortung dieser Parteien auf der Links-Rechts-Skala, entscheidenden Einfluss auf die Regierungspolitik ausübt (Castles 1982; Schmidt 1996a, 1996b; 2000: 378-389). Diese These geht davon aus, dass christdemokratische, wirtschaftsliberale oder sozialdemokratische Parteien gut unterscheidbare Programme vertreten, weil sie die Interessen verschiedener Wählergruppen vertreten, und dass sich diese unterschiedlichen parteipolitischen Programmatiken in der Regierungspraxis auch in verschiedenen Politikergebnissen bemerkbar machen.

Entsprechend den Annahmen dieser These neigen sozialdemokratische Parteien in der Wirtschafts- und Sozialpolitik (und damit auch im Arbeitsrecht) zu mehr Staatsintervention und einer arbeitnehmerfreundlicheren Politik, während christdemokratische, konservative und vor allem wirtschaftsliberale Parteien auf staatliche Zurückhaltung zu Gunsten der freien Entfaltung der Marktkräfte setzen und insgesamt eine wirtschaftsfreundliche Politik verfolgen. Aus dieser Sicht müssten also Mitte-Links-Regierungen arbeitsrechtliche EU-Richtlinien, deren Ziel die Verbesserung von Arbeitnehmerrechten ist, wesentlich eher unterstützen und diese daher besser umsetzen als konservativ-liberale Regierungen. Auch wenn meine Fallstudien die grundsätzliche Stoßrichtung der Parteiendifferenz-These durchaus bestätigen, legen die hier vorgestellten Ergebnisse ei- 
Tabelle 2: Arbeitsrechtliche Grundpräferenzen unterschiedlicher Parteigruppierungen

\begin{tabular}{|c|c|c|c|}
\hline & $\begin{array}{l}\text { gegen staatliche } \\
\text { Intervention }\end{array}$ & $\begin{array}{l}\text { für staatliche } \\
\text { Intervention }\end{array}$ & $\begin{array}{c}\text { Realbeispiele } \\
\text { in den 1990er Jahren }\end{array}$ \\
\hline $\begin{array}{l}\text { wirtschaftsliberale } \\
\text { Parteien }\end{array}$ & $X$ & & $\begin{array}{l}\text { FDP (D), VVD (NL), } \\
\text { Konservative (GB), PD (IRL) }\end{array}$ \\
\hline $\begin{array}{l}\text { Christdemokraten } \\
\text { und Konservative }\end{array}$ & $\leftarrow-$ & & $\begin{array}{l}\text { CDU (D), CDA (NL), } \\
\text { FF (IRL), FG (IRL) }\end{array}$ \\
\hline $\begin{array}{l}\text { "moderne“ } \\
\text { Sozialdemokratie }\end{array}$ & $\leftarrow-$ & $\longrightarrow$ & $\begin{array}{l}\text { PvdA (NL), } \\
\text { Labour Party (GB) }\end{array}$ \\
\hline $\begin{array}{l}\text { "traditionelle“ } \\
\text { Sozialdemokratie }\end{array}$ & & $X$ & $\begin{array}{l}\text { SPD (D), } \\
\text { Labour Party (IRL) }\end{array}$ \\
\hline
\end{tabular}

nen differenzierteren Zusammenhang zwischen parteipolitischer Ausrichtung der Regierung und Umsetzungsqualität nahe.

Erstens wurden parteipolitische Differenzen nicht in allen von mir analysierten Fällen tatsächlich aktiviert. Inwiefern es zu einer Links-Rechts-Politisierung kommt, hängt dabei von verschiedenen nationalen Kontextbedingungen ab. Es ist jedenfalls nicht die Höhe des Anpassungsbedarfs, die dafür entscheidend ist. Vielmehr geht es um die betroffenen Regelungsmaterien und inwiefern diese mit bestimmten parteipolitischen Positionen der Regierungen zusammenpassen. Dies kann aber von Fall zu Fall höchst unterschiedlich sein. Die Reaktion kann unter anderem davon abhängig sein, wie sich die nötigen Reformen politisch vermarkten lassen, wie sie zu den aktuellen politischen Themenkonjunkturen passen oder welchen generellen Stellenwert die Erfüllung europarechtlicher Verpflichtungen innerhalb der Regierung genießt.

Zweitens zeigen die Fallstudien, dass man bei der Zuordnung einzelner Parteien zu bestimmten „Parteifamilien“ differenziert vorgehen muss. Aus den hier untersuchten Fällen und dem darin beobachteten Verhalten verschiedener Regierungsparteien können dabei grob vereinfacht vier unterschiedliche Gruppen von Parteien mit jeweils unterschiedlichen arbeitsrechtlichen Grundpräferenzen abgeleitet werden (siehe Tabelle 2).

Parteien mit einem klar wirtschaftsliberalen Profil wie die deutsche FDP oder die britische Konservative Partei begegnen arbeitsrechtlichen Regelungen grundsätzlich mit Skepsis, während von "traditionellen" sozialdemokratischen Parteien wie der irischen Labour Party eine grundsätzliche Unterstützung von Maßnahmen zu erwarten ist, welche die Verbesserung von Arbeitnehmerrechten zum Ziel haben. Zwischen diesen beiden Extremen lassen sich konservative und christdemokratische Parteien wie die deutsche CDU, die niederländische CDA oder auch die beiden großen irischen Volksparteien Fianna Fáil und Fine Gael einordnen, bei denen es eine grundsätzliche Bereitschaft gibt, gesetzliche Arbeitnehmerrechte zu schaffen und zu verbessern, gleichzeitig jedoch auch die Bedürfnisse der Wirtschaft in erheblichem Maße mit in die politische Strategiewahl einfließen. Wichtig ist, dass "moderne" sozialdemokratische Parteien wie die britische Labour Party unter Tony Blair oder die niederländische PvdA eine ähnliche Position einnehmen. Bei diesen Parteien sind arbeitnehmerfreundliche Grundhaltungen in erheblichem Maße durch Erwägungen der Wirtschaftsverträglichkeit potenzieller 
staatlicher Interventionen eingeschränkt (siehe dazu auch Seeleib-Kaiser 2002). ${ }^{10}$ Daher ist es möglich, dass Regierungen, die von solchen Parteien geführt werden, im Zuge von Deregulierungs- und Flexibilisierungsbestrebungen ebenfalls Widerstand gegen arbeitsrechtliche Regelungen leisten, wie es etwa bei der Umsetzung der Jugendarbeitsschutz-Richtlinie in den Niederlanden der Fall war (Treib 2002).

Während die konkrete Haltung der beiden letztgenannten Gruppierungen gegenüber arbeitsrechtlichen Interventionen von verschiedenen Kontextfaktoren wie insbesondere der Stärke unterschiedlicher Parteiflügel oder auch den zu erwartenden Kosten abhängt, zeigt sich ein deutlicher Unterschied zwischen modernen Sozialdemokraten und christlich-konservativen Parteien im Hinblick auf gleichstellungspolitische Fragen. So sind christdemokratische und konservative Parteien im Allgemeinen eher einem traditionellen Bild der beruflichen und familiären Rollen von Frauen und Männern verpflichtet und bringen daher wenig Unterstützung für gleichstellungspolitische Initiativen auf. Dagegen spricht die säkulare Herkunft von sozialdemokratischen Parteien, egal ob wirtschaftspolitisch eher "traditionell“ oder eher „modern“ ausgerichtet, dafür, dass diese Parteien gleichstellungspolitischen Maßnahmen aufgeschlossener gegenüber stehen.

Schließlich muss drittens beachtet werden, dass der Effekt unterschiedlicher Parteikonstellationen auf das Umsetzungsergebnis hinsichtlich der inhaltlichen und zeitlichen Dimension differenziert werden muss. Wie gezeigt, führte parteipolitische Ablehnung häufig zu Verschleppung der Umsetzung oder aber auch zu inhaltlich inkorrekter Anpassung. Parteipolitische Unterstützung wirkte sich allerdings unterschiedlich auf die inhaltliche und die zeitliche Dimension aus: Die aktive Förderung von Richtlinien durch Mitte-Links-Regierungen hatte tendenziell einen positiven Effekt auf die Substanz des Umsetzungsresultats und führte mitunter sogar zur Überimplementation. Kam es allerdings zu solchen Fällen von Überimplementation, so barg dies die Gefahr zeitlicher Verzögerungen aufgrund von Konflikten über die hinzugefügten Materien.

\section{Schlussfolgerungen}

Der vorliegende Beitrag befasste sich mit der Frage, welche Faktoren zu einer korrekten und pünktlichen Umsetzung europäischer Richtlinien beitragen und was man daraus für die prinzipielle Steuerungsfähigkeit der supranationalen Politik lernen kann. Auf der theoretischen Ebene setzte sich das Papier mit der in der aktuellen Forschung häufig vertretenen Misfit-These auseinander, der gemäß Qualität und Schnelligkeit der nationalen Anpassung an EU-Vorschriften im Wesentlichen durch das Ausmaß der Passgenauigkeit zwischen europäischen Vorgaben und den bestehenden nationalen $\mathrm{Ar}$ rangements bestimmt wird.

Die präsentierten empirischen Ergebnisse aus einer Studie zur Umsetzung von sechs arbeitsrechtlichen Richtlinien in vier Ländern machten deutlich, dass ein exklusiver Blick auf die strukturelle Übereinstimmung zwischen europäischen Anforderungen

10 Die aktuelle Debatte um die „Agenda 2010“ deutet darauf hin, dass sich auch die deutsche SPD momentan in einer Phase der programmatischen Neuorientierung von einer "traditionellen“ zu einer „modernen“ sozialdemokratischen Partei befindet. 
und existierenden nationalen Regelungen nur sehr begrenzt Aufschluss über Art und Zeitpunkt der beobachteten Anpassungen geben konnte. Ich plädiere daher für eine Perspektive, welche die unterstützenden oder opponierenden Haltungen politischer Akteure stärker in den Mittelpunkt der Analyse rückt. Innerhalb dieses Rahmens habe ich mich in diesem Beitrag darauf konzentriert, die wichtige Rolle der Parteipolitik bei der Umsetzung europäischer Richtlinien zu verdeutlichen. Mein zentrales Argument lautet, dass nationale Regierungen bei der Umsetzung nicht als reine Verteidiger des Status quo agieren, sondern dass ihre Reaktionen ganz wesentlich von ihren parteipolitisch determinierten Präferenzen abhängen.

Die vorgestellten empirischen Beispiele haben gezeigt, dass es durch parteipolitischen Widerstand auch bei geringem Anpassungsbedarf zu gezielter Verschleppung der Anpassung oder zu bewusster Falschumsetzung kommen kann. Aktive parteipolitische Unterstützung trug dagegen auch bei hohem Anpassungsbedarf zu zügiger und korrekter Umsetzung bei und führte in einigen Fällen sogar zu Überimplementation. Insgesamt wurde dabei deutlich, dass die Anpassung an die untersuchten arbeitsrechtlichen Richtlinien tendenziell eher bei Mitte-Rechts-Regierungen auf explizite Ablehnung stieß, während eine aktive Unterstützung vor allem bei Mitte-Links-Regierungen zu beobachten war.

Dieser Befund deckt sich jedoch nur teilweise mit den Erwartungen der Parteiendifferenz-These. Meine Ergebnisse weisen vielmehr auf einen differenzierten Zusammenhang zwischen parteipolitischer Ausrichtung der Regierung und Umsetzungsperformanz hin. Erstens werden parteipolitische Differenzen nur unter bestimmten Umständen aktiviert. Zweitens ist nur bei wirtschaftsliberalen Parteien einerseits und „traditionellen“ sozialdemokratischen Parteien andererseits mit klarer Ablehnung oder Befürwortung arbeitsrechtlicher Reformen zu rechnen. Die Haltungen christdemokratischer Parteien und auch „moderner“ sozialdemokratischer Parteien sind dagegen sowohl durch arbeitnehmerfreundliche als auch in hohem Maße durch wirtschaftsfreundliche Erwägungen bestimmt. Drittens schließlich muss der Effekt unterschiedlicher parteipolitischer Konstellationen hinsichtlich der inhaltlichen und der zeitlichen Dimension des Umsetzungsresultats differenziert werden.

Die analysierten Fälle zeigen also, dass parteipolitisch definierte Interessen von Regierungen neben dem strukturellen „fit“ oder „misfit“ mit in den Blick genommen werden müssen, wenn man Erfolg oder Scheitern der Umsetzung europäischer Richtlinien erklären will. Parteipolitische Präferenzen fungieren als eine Art „Brille“, durch die Regierungen den Anpassungsbedarf einer Richtlinie betrachten und dabei je nach Übereinstimmung oder Widerspruch mit diesen Präferenzen beurteilen. Es kommt also nicht auf den durch eine Richtlinie erzeugten Anpassungsbedarf an sich an, sondern auf die Bewertung der geforderten Anpassungen vor dem Hintergrund parteipolitischer Positionen. Wenn die Anforderungen einer Richtlinie bei dieser Beurteilung entweder explizite parteipolitische Unterstützung oder Ablehnung hervorrufen, so ist das eigentliche Ausmaß des „misfit" von nachrangiger Bedeutung.

Diese Erkenntnisse weisen zwar darauf hin, dass die Bedeutung der Passgenauigkeit zwischen europäischen Vorgaben und nationalen Regelungsstrukturen deutlich geringer ist als von den Anhängern der Misfit-These propagiert. Dennoch gehört dieser Faktor von nun an nicht gänzlich auf den sprichwörtlichen „Müllhaufen der (Theorie-)Ge- 
schichte“. Erstens hat die absolute Höhe des Anpassungsbedarfs sicherlich einen Einfluss auf die Intensität parteipolitischer Ablehnung - der Widerstand der Konservativen Regierung Großbritanniens gegen die Umsetzung der Arbeitszeit-Richtlinie war aufgrund der massiven Anpassungskosten wesentlich stärker als etwa die Opposition der deutschen CDU/CSU-FDP-Regierung gegen die relativ moderaten Anforderungen der Elternurlaubs-Richtlinie. Und zweitens wird parteipolitische Unterstützung oder Ablehnung nicht immer auch tatsächlich aktiviert. Ob es zu einer solchen Politisierung kommt, ist von verschiedenen Kontextbedingungen abhängig. Eine wichtige Rolle spielen dabei sicherlich Dinge wie aktuelle politische Themenkonjunkturen, die politische Vermarktungsmöglichkeit von Ablehnung bzw. Unterstützung der infrage stehenden Regelungen oder auch der generelle Stellenwert von europarechtskonformem Verhalten innerhalb der Regierung.

Darüber hinaus gibt es mit Sicherheit politikfeldspezifische Unterschiede in der absoluten Bedeutung parteipolitischer Faktoren. Die zentrale Rolle der Parteipolitik in den hier untersuchten Fällen ist sicherlich nicht zuletzt auf das allgemein hohe Politisierungsniveau im Bereich arbeitsrechtlicher Regulierung zurückzuführen. Dies kann sich in anderen Politikfeldern ganz anders darstellen. So scheinen politische und administrative Akteure in Bereichen wie der Regionalpolitik in sehr viel stärkerem Maße offen für Policy-Lernen zu sein (siehe etwa Kohler-Koch 2002). Die Effektivität solcher supranationalen Überzeugungsstrategien hatte aber in den hier untersuchten Fällen enge Grenzen. Regierungen wie das britische Tory-Kabinett unter John Major, die arbeitsrechtlichen EU-Maßnahmen aus politisch-ideologischen Gründen ablehnend gegenüber stehen, lassen sich von deren politischer Sinnhaftigkeit ganz offensichtlich auch nicht durch gute Argumente überzeugen - Policy-Lernen scheint nur dann zu greifen, wenn fundamentale parteipolitische Interessen dem nicht entgegen stehen.

Auf ähnliche Weise legen die Beiträge der "Misfit-Schule“ nahe, dass in der EUUmweltpolitik, mit der sich diese Beiträge schwerpunktmäßig befasst haben, die parteipolitische Links-Rechts-Logik offenbar weniger stark ausgeprägt ist als die auf strukturelle Inkompatibilitäten abstellende Logik von „fit" und „misfit“. Aus diesem Grund plädiere ich keineswegs dafür, den Faktor „Anpassungsbedarf" in der EU-Implementationsforschung ad acta zu legen. Meine Erkenntnisse weisen aber daraufhin, dass es daneben noch andere wichtige Einflussgrößen gibt, von denen sich die Parteipolitik in den von mir untersuchten Fällen als besonders wichtig erwiesen hat. Die weitere Forschung wird zeigen müssen, welche Bedingungen dafür verantwortlich sind, dass manchmal die eine und manchmal die andere Logik zum Tragen kommt.

Meine Resultate werfen ein neues Licht auf die prinzipielle Steuerungs- und Durchsetzungsfähigkeit der supranationalen Politik. Zunächst ist festzuhalten, dass es durchaus ernstzunehmende Implementationsdefizite innerhalb der EU gibt. Ähnlich wie dies bereits von der frühen Implementationsforschung für die nationale Ebene festgestellt wurde (siehe z.B. Pressman/Wildavsky 1973; Mayntz 1980, 1983), ist auch in der Europäischen Union die faktische Durchsetzung einmal getroffener politischer Entscheidungen eine äußerst problematische Angelegenheit. Es dauert häufig mehrere Jahre, bis Brüsseler Beschlüsse in den Mitgliedsstaaten auch tatsächlich wirksam werden. Und trotz der in den meisten Richtlinien angelegten Möglichkeiten zur flexiblen Anpassung der Vorgaben an nationale Gegebenheiten kommt auch der verbindliche Kern der ver- 
einbarten Regelungen mitunter nur unvollständig oder verfälscht bei den eigentlichen Adressaten an - ein Zustand, der viele Jahre fortbestehen kann, bevor die Europäische Kommission und der Europäische Gerichtshof durch entsprechende Interventionen für eine Durchsetzung des Gemeinschaftsrechts sorgen. Anders gesagt: Wenn die EU durch eine gewählte Regierung geführt würde, müsste diese schon mit einer ungewöhnlich langen Legislaturperiode ausgestattet werden, damit ihre Reformmaßnahmen eine gewisse Chance hätten, ihre Wirkung zu entfalten, bevor sie sich das nächste Mal dem Urteil der Wähler stellen müsste.

Des Weiteren resultiert aus der Analyse, dass einer erfolgreichen politischen Steuerung im europäischen Mehrebenensystem offenbar in wesentlich geringerem Maße strukturelle Hürden im Wege stehen, als dies von Teilen der aktuellen Forschung suggeriert wird. Aus den Ergebnissen der Misfit-Schule lässt sich ja letztlich nur der Schluss ableiten, dass die EU sich ambitionierter politischer Interventionen enthalten sollte, weil die dadurch erforderlich werdenden, weit reichenden Reformen auf der nationalen Ebene unweigerlich an den Beharrungskräften der nationalen Regelungsstrukturen scheitern müssen. Ein solcher Steuerungsverzicht scheint angesichts der hier präsentierten Ergebnisse jedoch weder notwendig noch besonders hilfreich zu sein. Selbst ambitionierte politische Interventionen der EU müssen nicht notwendigerweise an der Beharrungskraft nationaler Regelungsstrukturen scheitern. Die hohe strukturelle Diversität nationaler Regelungstraditionen in vielen Politikbereichen muss also nicht unbedingt eine Hürde für erfolgreiche Mindestharmonisierung sein, sofern entsprechender politischer Gestaltungswillen auf der nationalen Ebene existiert. Andererseits ergeben sich durch den politischen Eigenwillen nationaler Regierungen auch neue Steuerungsprobleme, die etwa dazu führen können, dass selbst weniger weit reichende Reformen am Widerstand nationaler Akteure scheitern.

Meine empirischen Fallstudien weisen jedenfalls darauf hin, dass man bei der Analyse der Politikgestaltung innerhalb der EU die politischen Kontexte in den Mitgliedsstaaten, und insbesondere den Einfluss der Parteipolitik auf das Regierungshandeln, wesentlich stärker in den Blick nehmen sollte, als dies bislang häufig der Fall war. Für ein Verständnis der Problemlösungsfähigkeit der EU ist ein gründliches Studium nationaler Wahlergebnisse, Koalitionsvereinbarungen und Parteiprogramme daher mindestens ebenso wichtig wie die Lektüre international vergleichender Handbücher über das Arbeits-, Umwelt- oder Verbraucherschutzrecht in den Mitgliedsstaaten.

\section{Literatur}

Anzinger, Rudolf, 1994: Arbeitszeitgesetz: Erfolgreich zum Abschluß gebracht, in: Bundesarbeitsblatt 6/1994, 5-9.

ArbuR, 1993: Keine Einigung über EG-Arbeitszeitrichtlinie, in: Arbeit und Recht 41(3), 79.

Beckmann, Petra/Kurtz, Beate, 2001: Erwerbstätigkeit von Frauen: Die Betreuung der Kinder ist der Schlüssel, IAB-Kurzbericht Nr. 10, Nürnberg, Institut für Arbeitsmarkt- und Berufsforschung.

Börzel, Tanja A., 2000a: Improving Compliance through Domestic Mobilisation? New Instruments and Effectiveness of Implementation in Spain, in: Knill, Christoph/Lenschow, Andrea (Hrsg.), Implementing EU Environmental Policy: New Directions and Old Problems. Manchester, 222-250. 
Börzel, Tanja A., 2000b: Why There Is No ,Southern Problem': On Environmental Leaders and Laggards in the European Union, in: Journal of European Public Policy 7, 141-162.

Castles, Francis G. (Hrsg.), 1982: The Impact of Parties: Politics and Policies in Democratic Capitalist States. London.

DiMaggio, Paul J./Powell, Walter W., 1991: Introduction, in: Powell, Walter W./DiMaggio, Paul J. (Hrsg.), The New Institutionalism in Organizational Analysis. Chicago, 1-38.

Duina, Francesco G., 1997: Explaining Legal Implementation in the European Union, in: International Journal of the Sociology of Law 25, 155-179.

Duina, Francesco G., 1999: Harmonizing Europe: Nation-States within the Common Market. Albany.

Duina, Francesco G./Blithe, Frank, 1999: Nation-States and Common Markets: The Institutional Conditions for Acceptance, in: Review of International Political Economy 6, 494-530.

Dürmeier, Silvia, 1999: Teilzeitarbeit im Kontext europäischer Kollektivvereinbarungen, DWP 99.01.04. Brussels, European Trade Union Institute [http://www.etuc.org/etui/publications/DWP/Dürmeier2.PDF].

Falkner, Gerda, 2000: EG-Sozialpolitik nach Verflechtungsfalle und Entscheidungslücke: Bewertungsmaßstäbe und Entwicklungstrends, in: Politische Vierteljahresschrift 41, 279-301.

Grande, EdgarlJachtenfuchs, Markus (Hrsg.), 2000: Wie problemlösungsfähig ist die EU? Regieren im europäischen Mehrebenensystem. Baden-Baden.

Gray, Margaret, 1998: A Recalcitrant Partner: The UK Reaction to the Working Time Directive, in: Barav, Ami/Wyatt, Derrick A. (Hrsg.), Yearbook of European Law 1997. Oxford, 324-362.

Günther, Horst, 1993: Gemeinsamer Standpunkt verabschiedet, in: Bundesarbeitsblatt 10, 17-20.

Hepple, Bob/Fredman, Sandra, 1992: Labour Law and Industrial Relations in Great Britain. Deventer.

Hornung-Draus, Renate, 1996: Abkommen zum Elternurlaub verabschiedet, in: Der Arbeitgeber 48(3), 62-63.

Immergut, Ellen M., 1998: The Theoretical Core of the New Institutionalism, in: Politics and Society $26,5-34$.

Knill, Christoph/Lenschow, Andrea, 1998: Coping with Europe: The Impact of British and German Administrations on the Implementation of EU Environmental Policy, in: Journal of European Public Policy 5, 595-614.

Knill, Christoph/Lenschow, Andrea, 1999: Neue Konzepte - alte Probleme? Die institutionellen Grenzen effektiver Implementation, in: Politische Vierteljahresschrift 40, 591-617.

Knill, Christoph/Lenschow, Andrea, 2000: Do New Brooms Really Sweep Cleaner? Implementation of New Instruments in EU Environmental Policy, in: Knill, Christoph/Lenschow, Andrea (Hrsg.), Implementing EU Environmental Policy: New Directions and Old Problems. Manchester, 251-282.

Knill, Christoph/Lenschow, Andrea, 2001: Adjusting to EU Environmental Policy: Change and Persistence of Domestic Administrations, in: Green Cowles, Maria/Caporaso, James/Risse, Thomas (Hrsg.), Transforming Europe: Europeanization and Domestic Change. Ithaca, 116-136.

Kohler-Koch, Beate, 2002: On Networks, Travelling Ideas, and Behavioural Inertia, in: Conzelmann, Thomas/Knodt, Michèle (Hrsg.), Regionales Europa - Europäisierte Regionen. Frankfurt a.M., 87-103.

Lewis, Jeffrey, 1998: Constructing Interests: The Committee of Permanent Representatives and Decision-Making in the European Union. Dissertation, University of Wisconsin-Madison.

Lörcher, Klaus, 1994: Die Arbeitszeitrichtlinie der EU, in: Arbeit und Recht 42(2), 49-51.

March, James G./Olsen, Johan P., 1989: Rediscovering Institutions: The Organizational Basis of Politics. New York.

Mayntz, Renate, 1980: Implementation politischer Programme. Königstein/Ts.

Mayntz, Renate, 1983: Implementation politischer Programme II: Ansätze zur Theoriebildung. Opladen.

Mendrinou, Maria, 1996: Non-Compliance and the European Commission's Role in Integration, in: Journal of European Public Policy 3, 1-22. 
Ministerrat, 1996: Entwurf eines Protokolls über die 1914. Tagung des Rates (Arbeit und Sozialfragen) am 29. März 1996. Brüssel, Rat der Europäischen Union.

Pierson, Paul, 2000: Increasing Returns, Path Dependence, and the Study of Politics, in: American Political Science Review 94, 251-267.

Pressman, Jeffrey L./Wildavsky, Aaron, 1973: Implementation: How Great Expectations in Washington Are Dashed in Oakland: Or, Why It's Amazing that Federal Programs Work at All this Being a Saga of the Economic Development Administration as Told by Two Sympathetic Observers Who Seek to Build Morals on a Foundation of Ruined Hopes. Berkeley.

Prondzynski, Ferdinand von/Richards, Wendy, 1994: Ireland, European Employment and Industrial Relations Glossary. London.

Rhodes, Martin, 1995: A Regulatory Conundrum: Industrial Relations and the Social Dimension, in: Leibfried, Stephan/Pierson, Paul (Hrsg.), European Social Policy: Between Fragmentation and Integration. Washington, DC, 78-122.

Scharpf, Fritz W., 1985: Die Politikverflechtungs-Falle: Europäische Integration und deutscher Föderalismus im Vergleich, in: Politische Vierteljahresschrift 26, 323-356.

Scharpf, Fritz W., 1998: Die Problemlösungsfähigkeit der Mehrebenenpolitik in Europa, in: KohlerKoch, Beate (Hrsg.), Regieren in entgrenzten Räumen, PVS-Sonderheft 29. Opladen, 121-144.

Scharpf, Fritz W., 1999: Regieren in Europa: Effektiv und demokratisch? Frankfurt a.M.

Schmidt, Manfred G., 1996a: The Parties-Do-Matter-Hypothesis and the Case of the Federal Republic of Germany, in: German Politics 4, 1-21.

Schmidt, Manfred G., 1996b: When Parties Matter: A Review of the Possibilities and Limits of Partisan Influence on Public Policy, in: European Journal of Political Research 30, 155-183.

Schmidt, Manfred G., 2000: Demokratietheorien. Opladen.

Seeleib-Kaiser, Martin, 2002: Neubeginn oder Ende der Sozialdemokratie? Eine Untersuchung zur programmatischen Reform sozialdemokratischer Parteien und ihrer Auswirkung auf die Parteiendifferenzthese, in: Politische Vierteljahresschrift 43, 478-496.

Statistisches Bundesamt, 2002: Statistisches Jahrbuch für die Bundesrepublik Deutschland 2002. Wiesbaden.

Thelen, Kathleen/Steinmo, Sven, 1992: Historical Institutionalism in Comparative Politics, in: Steinmo, Sven/Thelen, Kathleen/Longstreth, Frank (Hrsg.), Structuring Politics: Historical Institutionalism in Comparative Analysis. Cambridge, 1-32.

Treib, Oliver, 2002: Europäische Vorgaben, nationaler Anpassungsbedarf und seine politische Verarbeitung: Eine ländervergleichende Studie über die Umsetzung arbeitsrechtlicher EU-Richtlinien unter besonderer Berücksichtigung von Deutschland, den Niederlanden, Großbritannien und Irland. Dissertation, Universität zu Köln.

Tsebelis, George, 1995: Decision Making in Political Systems: Veto Players in Presidentialism, Parliamentarism, Multicameralism and Multipartism, in: British Journal of Political Science 25, 289-325.

Van der Heijden, Paul F., 1999: The Netherlands, in: Gold, Michael/Weiss, Manfred (Hrsg.), Employment and Industrial Relations in Europe. Den Haag, 143-166.

Weiss, Manfred, 1995: Labour Law and Industrial Relations in Germany. Deventer.

Zohlnhöfer, Reimut, 2001: Die Wirtschaftspolitik der Ära Kohl: Eine Analyse der Schlüsselentscheidungen in den Politikfeldern Finanzen, Arbeit und Entstaatlichung, 1982-1998. Opladen. 